\title{
Analysis of Maxwell's Equations. Cosmic Magnetism
}

\author{
Vladimir S. Netchitailo \\ Biolase Inc., Irvine, CA, USA \\ Email:v.netchitailo@sbcglobal.net
}

How to cite this paper: Netchitailo, V.S. (2018) Analysis of Maxwell's Equations. Cosmic Magnetism. Journal of High Energy Physics, Gravitation and Cosmology, 4, 1-7. https://doi.org/10.4236/jhepgc.2018.41001

Received: October 13, 2017

Accepted: November 14, 2017

Published: November 17, 2017

Copyright $\odot 2018$ by author and Scientific Research Publishing Inc. This work is licensed under the Creative Commons Attribution International License (CC BY 4.0).

http://creativecommons.org/licenses/by/4.0/

\section{c) (i) Open Access}

\begin{abstract}
According to Hypersphere World-Universe Model, dark matter particles DIRACs are magnetic dipoles consisting of two Dirac's monopoles. We conclude that DIRACs are the subject of Maxwell's equations. So-called "auxiliary" magnetic field intensity $\mathbf{H}$ is indeed current density of magnetic dipoles. The developed approach to magnetic field can explain a wealth of discovered phenomena in Cosmic Magnetism: a dark magnetic field, the large-scale structure of the Milky Way's magnetic field, and other magnetic phenomena which are only partly related to objects visible in other spectral ranges.
\end{abstract}

\section{Keywords}

Hypersphere World-Universe Model, Maxwell's Equations, Dirac's

Monopole, Magnetic Dipole, Magnetic Field Intensity, Magnetic Dipoles

Current Density, Cosmic Magnetism, Dark Magnetic Field

\section{Introduction}

Maxwell's equations (ME) form the foundation of classical electrodynamics. The value of ME is even greater once J. Swain showed that linearized general relativity admits a formulation in terms of gravitoelectric and gravitomagnetic fields that closely parallel the description of the electromagnetic field by Maxwell's equations [1].

H. Thirring pointed out this analogy in his "On the formal analogy between the basic electromagnetic equations and Einstein's gravity equations in first approximation" paper published in 1918 [2]. It allows us to use formal analogies between Electromagnetism and Gravitoelectromagnetism [3] proposed by Oliver Heaviside in 1893. Hypersphere World-Universe Model is based on Maxwell's equations [3]. 


\section{Analysis of Maxwell's Equations}

Maxwell's equations vary with the unit system used. We will not rewrite well-known equations, but only provide the relationships between electromagnetic quantities used in ME. Table 1 gives the definitions of these quantities in SI units. Electrodynamic constant $c$ is defined as the ratio of the absolute electromagnetic unit of charge to the absolute electrostatic unit of charge [3].

In $\mathrm{ME}$, there are two physical sources: the total electric charge density $\rho$ and the total electric current density $J$. According to ME, there are two measurable physical characteristics: energy density $\rho_{E}$ and energy flux density $\boldsymbol{J}_{E}$.

It is interesting to proceed with Maxwell's equations for Electromagnetism and Gravitoelectromagnetism when the physical sources are energy density $\rho_{E}$ and energy flux density $\boldsymbol{J}_{E}$ which coincide with the same measurable physical characteristics.

To apply ME, it is necessary to specify the constitutive relations:

$$
\begin{aligned}
\boldsymbol{D} & =\varepsilon_{0} \boldsymbol{E} \\
\boldsymbol{H} & =\frac{1}{\mu_{0}} \boldsymbol{B}
\end{aligned}
$$

The original equations given by Maxwell included Ohms law in the following form:

$$
\boldsymbol{E}=\rho_{r} \boldsymbol{J}
$$

where $\rho_{r}$ is the resistivity. In ME we can take the value of $\rho_{r}$ to equal

$$
\rho_{r}=Z_{0} \times a
$$

Where $\alpha$ is the basic unit of size: $a=2 \pi a_{0}, a_{0}$ being the classical electron radius. The total electric charge $Q$ enclosed in the volume $V$ is the volume integral over $V$ of the electric charge density $\rho$ :

$$
Q=\iiint \rho \mathrm{d} v
$$

The net electric current $I$ is the surface integral of the electric current density $J$ passing through a fixed surface $S$ :

$$
I=\iint \boldsymbol{J} \mathrm{d}
$$

Table 1. Electromagnetism.

\begin{tabular}{ccc}
\hline Charge & Impedance of Electromagnetic Field & Magnetic Flux \\
\hline$q, C$ & $Z_{0}=\sqrt{\mu_{0} / \varepsilon_{0}}=\mu_{0} c, \Omega$ & $\phi, W b$ \\
Electric Current & Magnetic Constant & Electric Potential \\
$I, A$ & $\mu_{0}, \mathrm{Hm}^{-1}$ & $\mathrm{U}, \mathrm{V}$ \\
Magnetic Field Intensity & Electric Constant & Electric Field \\
$\boldsymbol{H}, \mathrm{Am}^{-1}$ & $\varepsilon_{0}=\left(\mu_{0} \mathrm{C}^{2}\right)^{-1}, \mathrm{Fm}^{-1}$ & $\boldsymbol{E}, \mathrm{Vm}^{-1}$ \\
Electric Flux Density & Electrodynamic Constant & Magnetic Flux Density \\
$\boldsymbol{D}, \mathrm{Cm}^{-2}$ & $\boldsymbol{c}, \mathrm{ms}^{-1}$ & $\boldsymbol{B}, \mathrm{Wbm}^{-2}$
\end{tabular}


Electric potential $U$ is the line integral along a curve $L$ of the electric field $E$ :

$$
U=\int \boldsymbol{E} \mathrm{d}
$$

The magnetic flux is the surface integral of the magnetic flux density $\boldsymbol{B}$ passing through a fixed surface $S$ :

$$
\phi=\iint \boldsymbol{B} \mathrm{d} \boldsymbol{s}
$$

We emphasize that all these quantities in ME can be calculated based on physical sources $\rho$ and $J$. There are two auxiliary field quantities:

$$
\begin{gathered}
\boldsymbol{D}=\varepsilon_{0} \boldsymbol{E}+\boldsymbol{P} \\
\boldsymbol{H}=\frac{1}{\mu_{0}} \boldsymbol{B}-\boldsymbol{M}
\end{gathered}
$$

The quantities $\boldsymbol{P}$ and $\boldsymbol{M}$ represent the macroscopically averaged electric dipole and magnetic dipole moment densities of the material medium in the presence of applied fields. Analysis of ME in which all quantities introduced above are arbitrary functions of space and time has been done in literature (see, for example [4] [5]).

Maxwell's equations posit that there is electric charge, but no magnetic charge (magnetic monopole) in the World. K. Brown has this to say about magnetic dipole fields [6]:

There do, however, exist what appear to be magnetic dipoles, analogous to electric dipoles consisting of adjacent positive and negative electric charges. It might seem as if the existence of magnetic dipoles is indirect proof of the existence of individual magnetic charges, assuming the only way to produce a dipole field is by juxtaposing two oppositely charged magnetic monopoles. However, there is an alternative way of creating a magnetic "dipole" field without actually using magnetic charges. The alternative is an electric current loop. It can be shown that a circular loop of electric current produces a magnetic field that is (outside a spherical region enclosing the loop) nearly identical to the field of two adjacent and oppositely charged magnetic monopoles (if such things existed). So, we have two possible classical models for the source of "magnetic dipole" fields, one based on the juxtaposition of two oppositely charged magnetic monopoles, and one based on a loop of electric current. These two models might be called Coulombic and Amperean dipoles respectively.

M. Mansuripur compared two versions of the Poynting vector $S=\frac{1}{\mu_{0}} \boldsymbol{E} \times \boldsymbol{B}$ and $\boldsymbol{S}=\boldsymbol{E} \times \boldsymbol{H}$. He argues that the identification of one or the other of these Poynting vectors with the rate of flow of electromagnetic energy is intimately tied to the nature of magnetic dipoles and the way in which these dipoles exchange energy with the electromagnetic field. Hidden energy and hidden momentum can be avoided, however, if we adopt $\boldsymbol{S}=\boldsymbol{E} \times \boldsymbol{H}$ as the true Poynting vector, and also accept a generalized version of the Lorentz force law. He concludes that the identification of magnetic dipoles with Amperian current loops, 
while certainly acceptable within the confines of Maxwells macroscopic equations, is inadequate and leads to complications when considering energy, force, torque, momentum, and angular momentum in electromagnetic systems that involve the interaction of fields and matter [4].

$\mathrm{K}$. Brown emphasizes the difference between the $\boldsymbol{B}$ and the $\boldsymbol{H}$ fields. Outside any magnetic material, $\boldsymbol{B}$ and $\boldsymbol{H}$ are strictly proportional to each other, but inside magnetic material they are quite different. The potential energy density of a magnetic field is really $\frac{1}{2} \boldsymbol{B} \cdot \boldsymbol{H}$, and reduces to $\frac{1}{2 \mu_{0}} \boldsymbol{B}^{2}$ only outside of any magnetic material [6].

According to Hypersphere World-Universe Model (WUM), the Medium of the World consists of the following elementary particles [7]:

- Protons and electrons with mass $m_{p}$ and $m_{e}$ and electric charge $e$;

- Mass-varying neutrinos and photons;

- Dark Matter Particles (DMP) including fermions (neutralinos, WIMPs, and sterile neutrinos) and bosons:

o ELOPs with mass $\frac{2}{3} m_{e}$ that are electric dipoles of preons with electric charges $\frac{1}{3} e$. They represent the macroscopically averaged electric dipole moment density $\boldsymbol{P}$ of the Medium of the World with energy density about the proton energy density [7].

o DIRACs with mass $m_{0}$ which are magnetic dipoles of Dirac's monopoles with magnetic charges $\mu=\frac{e}{2 \alpha}$, where $\alpha=\frac{m_{e}}{m_{0}} \quad\left(m_{0}\right.$ is the basic unit of mass $m_{0}=\frac{h}{a c}, h$ is Planck constant) [7].

In our opinion, DIRACs are the Coulombic magnetic dipoles as was discussed by K. S. Brown [6]. Their energy density in the Medium of the World is about the proton energy density [7]. They represent the macroscopically averaged magnetic dipole moment density $\boldsymbol{M}$ of the Medium in the presence of applied fields.

It is well-known that the dimension of the magnetic field intensity $[\boldsymbol{H}]=\mathrm{Am}^{-1}$. We can rewrite it in the following way:

$$
[\boldsymbol{H}]=\frac{C m}{m^{2} s}=\frac{\left[\boldsymbol{d}_{m}\right]}{m^{2} s}=\left[\boldsymbol{J}_{m}\right]
$$

where $\boldsymbol{d}_{m}$ is a magnetic dipole momentum. It looks like magnetic field intensity $H$ is, in fact, the current density $\boldsymbol{J}_{m}$ of magnetic dipoles $d_{m}$. In our opinion, the magnetic field intensity $H$ is not an "auxiliary" field quantity. On the contrary, it is a real magnetic field quantity. That is why $\boldsymbol{S}=\boldsymbol{E} \times \boldsymbol{H}$ is the true Poynting vector and $\frac{1}{2} \boldsymbol{B} \cdot \boldsymbol{H}$ is the true potential energy density of a magnetic field.

Let's calculate the value of magnetic dipole momentum $\boldsymbol{d}_{m}$. We'll start from the original equations. The Dirac's quantization equation introduces the mag- 
netic monopole:

$$
\frac{e^{2}}{4 \pi \varepsilon_{0}}=n \frac{h c}{4 \pi}
$$

where $n$ is an integer, and $e$ and $\mu$ are electromagnetic charges. Considering the following well-known equation

$$
\frac{e^{2}}{4 \pi \varepsilon_{0}}=\frac{\alpha h c}{2 \pi}
$$

for $n=1$ we obtain the minimum magnetic charge $\mu=\frac{e}{2 \alpha}$. Impedance of electromagnetic field $Z_{0}$ equals to

$$
Z_{0}=\frac{1}{\varepsilon_{0} c}=\frac{h}{e \mu}
$$

Using the equations for $Z_{0}$ and $\mu$ derived above, we obtain the magnetic parameter $\mu_{0}$ :

$$
\mu_{0}=\frac{h}{e \mu c}
$$

Using the constitutive relation

$$
\boldsymbol{B}=\mu_{0} \boldsymbol{H}=\mu_{0} \boldsymbol{J}_{m}
$$

we can express the magnetic flux with the following equation:

$$
\phi=\mu_{0} \iint \boldsymbol{J}_{m} \mathrm{~d} s=\mu_{0} I_{m}
$$

where $I_{m}$ is the current of magnetic dipoles $d_{m}$. Magnetic flux quantum $\phi_{0}$ can then be expressed as follows:

$$
\phi_{0}=\frac{h}{2 e}=\mu_{0} I_{0}=\frac{h}{e \mu c} \frac{\mu c}{2}
$$

and the quant of magnetic dipole current $I_{0}$ is:

$$
I_{0}=\frac{\mu c}{2}=\frac{\frac{\mu a}{2}}{t_{0}}
$$

where $t_{0}$ is the basic unit of time [7]:

$$
t_{0}=\frac{a}{c}
$$

It means that the magnetic flux $\phi$ is the current of the magnetic dipoles:

$$
d_{m}=\frac{\mu a}{2}
$$

which are DIRACs in WUM. DIRACs have negligible electromagnetic charges, since the separation between charges $\mu$ is very small $a / 2$. They do, however, possess a substantial magnetic dipole momentum $\boldsymbol{d}_{m}$. The same conclusion can be derived for ELOPs-electric dipoles made of two preons with charges $\frac{1}{3} e$.

To summarize, magnetic monopoles are not the subject of Maxwell's equa- 
tions; instead, magnetic dipoles DIRACs are. So-called "auxiliary" magnetic field intensity $H$ is indeed current density of magnetic dipoles.

\section{Cosmic Magnetism}

R. Beck and R. Wielebinski have this to say about Cosmic Magnetism:

Most of the visible matter in the Universe is ionized, so that cosmic magnetic fields are quite easy to generate and due to the lack of magnetic monopoles hard to destroy. Magnetic fields have been measured in or around practically all celestial objects, either by in-situ measurements of spacecrafts or by the electromagnetic radiation of embedded cosmic rays, gas or dust. The Earth, the Sun, solar planets, stars, pulsars, the Milky Way, nearby galaxies, more distant (radio) galaxies, quasars and even intergalactic space in clusters of galaxies have significant magnetic fields, and even larger volumes of the Universe may be permeated by "dark" magnetic fields [8].

In frames of WUM, the similarity of field patterns and flow patterns of the diffuse ionized gas [8] can be explained by the flow of DIRACs along with diffuse ionized gas. The large-scale structure of the Milky Way's magnetic field [9], a dark magnetic field [10] and other magnetic phenomena which are only partly related to objects visible in other spectral ranges [8] can be explained by flows of dark matter particles DIRACs. We believe that the developed approach to magnetic field can answer questions on the origin and evolution of magnetic fields such as their first occurrence in young galaxies, or the existence of large-scale intergalactic fields [8]. Hypersphere World-Universe Model can serve as a basis for Cosmic Magnetism.

\section{Acknowledgements}

Special thanks to my son Ilya Netchitailo who helped shape this paper.

\section{References}

[1] Swain, J.D. (2010) Gravitatomagnetic Analogs of Electric Transformers. arXiv: 1006.5754 .

[2] Thirring, H. (1918) On the Formal Analogy between the Basic Electromagnetic Equations and Einstein's Gravity Equations in First Approximation. Physikalische Zeitschrift, 19, 204.

[3] Netchitailo, V.S. (2016) 5D World-Universe Model. Gravitation. Journal of High Energy Physics, Gravitation and Cosmology, 2, 328. https://doi.org/10.4236/jhepgc.2016.23031

[4] Mansuripur, M. (2012) Nature of Electric and Magnetic Dipoles Gleaned from the Poynting Theorem and the Lorentz Force Law of Classical Electrodynamics. arXiv: 1208.0873

[5] Michon, G.P. (2017) Electromagnetism: Maxwell's Equations and Their SolutionsNumericana. http://www.numericana.com/answer/maxwell.htm

[6] Brown, K.S. (2017) Magnetic Dipoles. http://www.mathpages.com/home/kmath694/kmath694.htm 
[7] Netchitailo, V.S. (2017) Mathematical Overview of Hypersphere World-Universe Model. Journal of High Energy Physics, Gravitation and Cosmology, 3, 415. https://doi.org/10.4236/jhepgc.2017.33033

[8] Beck, R. and Wielebinski, R. (2013) Magnetic Fields in Galaxies. arXiv: 1302.5663. https://doi.org/10.1007/978-94-007-5612-0_13

[9] Han, J.L. (2003) The Large-Scale Magnetic Field Structure of Our Galaxy: Efficiently Deduced from Pulsar Rotation Measures. The Magnetized Interstellar Medium. Antalya, 8-12 September 2003.

[10] Pitkanen, M. (2015) "Invisible Magnetic Fields" as Dark Magnetic Fields.. http://matpitka.blogspot.com/2015/09/invisible-magnetic-fields-as-dark.html?m=0 